TRANSACTIONS OF THE

AMERICAN MATHEMATICAL SOCIETY

Volume 360, Number 12, December 2008, Pages 6595-6609

S 0002-9947(08)04502-9

Article electronically published on July 28, 2008

\title{
MAXIMAL OPERATORS OF TREE MARTINGALE TRANSFORMS AND THEIR MAXIMAL OPERATOR INEQUALITIES
}

\author{
TONG-JUN HE AND YI SHEN
}

\begin{abstract}
In this paper we define maximal operators for tree martingale transforms in $U M D$ spaces and prove inequalities for them by using the $U M D$ property.
\end{abstract}

\section{INTRODUCTION}

Tree martingales have been studied by Schipp, Fridli, Weisz, Young, and others, but there are many open problems. In the 1980s, some inequalities relative to tree martingales were verified by Schipp and Fridli [6], 13. For $2<p<\infty$, a maximal inequality as well as Burkholder-Gundy's inequality is shown, and is extended to all $1<p<\infty$ for a regular stochastic basis by their works. Moreover, using the results on tree martingales, Schipp [12] and Gosselin [7] proved that for an arbitrary Vilenkin system and for $f \in L_{p}$, the Vilenkin-Fourier series of $f$ converges in the $L_{p}$ norm to itself. However, because of the fact that a tree martingale transform cannot be defined as a one-parameter martingale, similarly, stopping times cannot be introduced for tree martingales. Schipp and Fridli [13 obtained some results using convexity methods. The question is: Are there efficient ways to overcome these difficulties? Here we study this problem. In our paper, we define a new transform operator of vector-valued tree martingales by a linear operator. This tree martingale transform operator is not different from the one-parameter martingale transform in [3. Then we try to investigate this tree martingale transform operator by using the $U M D$ property.

$U M D$ spaces (unconditional martingale differences property) were studied by Burkholder [4- [5], Bourgain 2] and others. Burkholder, McConnell, and Bourgain proved that martingales with values in a Banach space $\mathbf{X}$ having the property of good martingale transforms is equivalent to the space $\mathbf{X} \in U M D$. We use this property here to study tree martingales with values in a $U M D$ space $\mathbf{X}$. This gives a simpler approach to tree martingales as we show here. It is still necessary to construct some useful convex functions and concave functions that we shall use in our proofs of our main theorems.

During the past ten years, relatively few people studied tree martingales. In [8, we verified that some scalar-valued tree martingale inequalities held, and in

Received by the editors August 28, 2006 and, in revised form, March 1, 2007.

2000 Mathematics Subject Classification. Primary 60G46, 46B09.

Key words and phrases. Tree martingale, UMD spaces, maximal operator.

The authors were partially supported by NSFC Grant: 60574025 .

(C)2008 American Mathematical Society 
9]-10], we investigated vector-valued tree martingales and some inequalities for them. In this paper, we shall discuss maximal operators of $\mathbf{X}$-valued tree martingale transforms and show that they are norm-bounded in $L_{p}(\mathbf{X})$ provided $\mathbf{X}$ is a $U M D$ space.

\section{Preliminaries And Definitions}

Let $\mathbf{T}$ be a countable, upward directed index set with respect to the partial ordering $\leq$ satisfying the following two conditions:

(1) for every $t \in \mathbf{T}$, the set $\mathbf{T}^{t}:=\{s \in \mathbf{T}: s \leq t\}$ is finite;

(2) for every $t \in \mathbf{T}$, the set $\mathbf{T}_{t}:=\{s \in \mathbf{T}: t \leq s\}$ is linearly ordered.

Thus $\mathbf{T}$ is a tree and every nonempty subset of $\mathbf{T}$ has at least one minimum. For every element $t \in \mathbf{T}$, the minimum element of the set $\mathbf{T}_{t} \backslash\{t\}$ is denoted by $t^{+}$; it is the succeeding element of $t$. A tree $\mathbf{T}$ is also a special partially ordered set with respect to the partial ordering $\leq$.

Let $(\Omega, \mathcal{F}, P)$ be a complete probability space, and $\mathcal{F}=\left(\mathcal{F}_{t}, t \in \mathbf{T}\right)$ be a family of nondecreasing sub- $\sigma$-algebras of $\mathcal{F}$ with respect to the partial ordering $\leq$. Then $\left(\mathcal{F}_{s}, s \in \mathbf{T}_{t}\right)$ can be linearly ordered, where $\mathcal{F}=\sigma\left(\bigcup_{t \in \mathbf{T}} \mathcal{F}_{t}\right)$. For simplicity, we assume that if $t=s^{+}=r^{+}$, then $\mathcal{F}_{s^{+}}=\mathcal{F}_{r^{+}}$. This common $\sigma$-algebra will be denoted by $\mathcal{F}_{t}^{-}$. Throughout this paper, unless otherwise stated, we let $E_{t}$ be the conditional expectation operator with respect to $\mathcal{F}_{t},(\mathbf{X},\|\|$.$) be a Banach space,$ and $L_{1}(\mathbf{X})$ be the space of Bochner integrable measurable functions. The indicator function of a set $\mathbf{A}$ is denoted by $\chi_{\mathbf{A}}$.

In the tree case, we work with projections instead of the conditional expectation operators. Let $\left(\phi_{t}, t \in \mathbf{T}\right)$ be a family of complex-valued measurable functions with $|\phi|=1$. For every $t \in \mathbf{T}$, set

$$
P_{t} f=\phi_{t} E_{t}\left(f \bar{\phi}_{t}\right), \quad f \in L_{1}(\mathbf{X}), \mathbf{t} \in \mathbf{T} .
$$

Then $\left(P_{t}, t \in \mathbf{T}\right)$ is a family of projections since $\left\|P_{t}\right\| \leq 1$ and $P_{t} \circ P_{t}=P_{t}(t \in \mathbf{T})$. It is clear that the conditional expectation operators are projections of the form (2.1).

Definition 2.1. Let $\left(P_{t}, t \in \mathbf{T}\right)$ be a family of projections as defined in (2.1). Then the family of $\left(\mathcal{F}_{t}, P_{t}: t \in \mathbf{T}\right)$ is called a tree basis if

(1) for every $f \in L_{1}(\mathbf{X})$ and $s \leq t(s, t \in \mathbf{T}), P_{t} f=\phi_{s} E_{t}\left(f \bar{\phi}_{s}\right)$;

(2) for every pair of incomparable $s, t$ in $\mathbf{T}, P_{t} P_{s}=0$.

Note that for any comparable $s, t \in \mathbf{T}$, if $s \leq t$ implies $P_{t} P_{s}=P_{s} P_{t}=P_{s}$, which follows from the previous (1), then we can proceed to define an $\mathbf{X}$-valued tree martingale.

Definition 2.2. We say that a family $f=\left(f_{t}, t \in \mathbf{T}\right)$ of $\mathbf{X}$-valued integrable functions is an $\mathbf{X}$-valued tree martingale if $s \leq t$ implies $P_{s} f_{t}=f_{s}$.

Clearly, the martingale with respect to a linearly ordered stochastic basis is the special tree martingale and for every $f \in L_{1}(\mathbf{X})$ the family of $\left(P_{t} f, t \in \mathbf{T}\right)$ is an $\mathbf{X}$-valued tree martingale. We always assume that for every $\mathbf{X}$-valued tree martingale there exists a distinguished minimal element $t_{0} \in \mathbf{T}$ such that $f_{t_{0}}=0$. It is important to note that for every $\mathbf{X}$-valued tree martingale $f=\left(f_{t}, t \in \mathbf{T}\right)$ and a fixed $t \in \mathbf{T}$, the sequence

$$
\left(\bar{\phi}_{t} f_{s}, s \in \mathbf{T}_{\mathbf{t}}\right)
$$


is a linearly ordered martingale. In fact, it follows from (1) of Definition 2.1 that if $t \leq r \leq s$, then $f_{s}=P_{s} f_{s}=\phi_{t} E_{s}\left(f_{s} \overline{\phi_{t}}\right)$; on the other hand, since $\left|\phi_{t}\right|^{2}=\phi_{t} \bar{\phi}_{t}=1$, so $\bar{\phi}_{t} f_{s}=E_{s}\left(f_{s} \bar{\phi}_{t}\right)$. Therefore, $\bar{\phi}_{t} f_{s}$ is $\mathcal{F}_{s}$-measurable and

$$
E_{r}\left(\bar{\phi}_{t} f_{s}\right)=E_{r}\left(f_{s} \bar{\phi}_{t}\right)=\bar{\phi}_{t} P_{r} f_{s}=\bar{\phi}_{t} f_{r}
$$

Then $\left(\bar{\phi}_{t} f_{s}, s \in \mathbf{T}_{\mathbf{t}}\right)$ is a linearly ordered martingale. We define the martingale difference of a family of $\mathbf{X}$-valued tree martingales $f=\left(f_{t}, t \in \mathbf{T}\right)$ by

$$
d_{t} f=f_{t^{+}}-f_{t} \quad(t \in \mathbf{T})
$$

obviously, for every $t \in \mathbf{T}, d_{t} f$ is a single function rather than a product of two functions. In the following, we introduce a linear operator that is more general than the martingale differences $\left(d_{t} f, t \in \mathbf{T}\right)$.

Definition 2.3. It is a family of linear operators $\pi=\left(\pi^{t}, t \in \mathbf{T}\right)$ satisfying the following conditions for all $f \in L_{1}(\mathbf{X})$ and $t \in \mathbf{T}$ :

(1) $P_{t^{+}}\left(\pi^{t} f\right)=\pi^{t} f$

(2) $P_{t}\left(\pi^{t} f\right)=0$;

(3) for every $\mathcal{F}_{t}$-measurable function $\xi$ one has $\pi^{t}(\xi f)=\xi \pi^{t} f$;

(4) $\left\|\pi^{t} f\right\| \leq R E_{t}\|f\|$, where the constant $R$ is independent of $t$ and $f$.

As mentioned earlier, an $\mathbf{X}$-valued tree martingale transform cannot be defined as a one-parameter martingale for some martingale unless it is already a one-parameter martingale. Similarly, stopping times cannot be introduced. Here we define an Xvalued tree martingale transform that is more general than the transform of a one-parameter martingale. In Definition 2.4, we define a maximal operator of an $\mathbf{X}$-valued tree martingale transform.

Definition 2.4. If $f=\left(f_{t}, t \in \mathbf{T}\right)$ is an $\mathbf{X}$-valued tree martingale, then these $\mathbf{X}$-valued tree martingale transforms are defined by

$$
\pi_{s} f=\sum_{t \leq r<s} \pi^{r}\left(d_{r} f\right) \quad\left(t \in \mathbf{T}, s \in \mathbf{T}_{t}\right),
$$

and the maximal operator of this $\mathbf{X}$-valued tree martingale transform is defined by

$$
\pi_{t} f=\sup _{s \in \mathbf{T}_{t}}\left\|\pi_{s} f\right\|, \quad \pi f=\sup _{t \in \mathbf{T}} \pi_{t} f .
$$

In the one-parameter martingale case, we see that $\pi$ is an operator of type $\left(L_{p}, L_{p}\right)(2 \leq p<\infty)$ from [15]; however, in the $\mathbf{X}$-valued tree martingale case, it is still unknown which type the operator $\pi$ is. This paper's aim is to show some results for the operator $\pi$ in the $\mathbf{X}$-valued tree martingale case. For this aim, we need to introduce a quasi-norm $\|\cdot\|_{\mathbf{M}^{p q}}$. Let $f=\left(f_{t}, t \in \mathbf{T}\right)$ be a family of $\mathcal{F}$-measurable functions (not necessarily a tree martingale) defined on the complete probability space $(\Omega, \mathcal{F}, P)$. For any $y \geq 0$, we set

$$
\nu_{y}^{f}=\inf \left\{t \in \mathbf{T}:\left\|f_{t}\right\|>y\right\} .
$$

Then it is easy to see that for every $t \in \mathbf{T}$,

$$
\left\{t \in \nu_{y}^{f}\right\}=\left\{\omega \in \Omega:\left\|f_{t}(\omega)\right\|>y,\left\|f_{s}(\omega)\right\| \leq y, \forall s<t\right\},
$$

where $s<t$ means that $s \leq t$ but $s \neq t$. Note that $\nu_{y}^{f}$ is generally a subset of $\mathbf{T}$, and if $\mathbf{T}$ is a totally ordered set, then $\nu_{y}^{f}$ is a stopping time; but if $\mathbf{T}$ is a partially 
ordered set, then $\nu_{y}^{f}$ isn't a stopping time, since for a fixed $y>0, \nu_{y}^{f}$ isn't one. Now, we can introduce the definition of quasi-norm $\|\cdot\|_{\mathbf{M}^{p q}}$ by $\nu_{y}^{f}$. For $0<p, q<\infty$, set

$$
\begin{aligned}
\|f\|_{\mathbf{M}^{p q}} & =\sup _{y>0} y\left(\int_{\Omega}\left(\sum_{t \in \mathbf{T}} \chi_{\left\{t \in \nu_{y}^{f}\right\}}\right)^{\frac{p}{q}}\right)^{\frac{1}{p}}, \\
\mathbf{M}^{p q} & =\left\{f=\left(f_{t}, t \in \mathbf{T}\right):\|f\|_{\mathbf{M}^{p q}}<\infty\right\},
\end{aligned}
$$

where $\chi_{\left\{t \in \nu_{y}^{f}\right\}}$ is the indicator function of the set $\left\{t \in \nu_{y}^{f}\right\}$.

Remark 2.5. Note that for each fixed family $f=\left(f_{t}, t \in \mathbf{T}\right)$, the quasi-norm $\|f\|_{\mathbf{M}^{p q}}$ is decreasing with $q$ increasing and is increasing with $p$ increasing. Therefore, the limit does exists as $q \rightarrow \infty$ and satisfies

$$
\|f\|_{\mathbf{M}^{p \infty}}=\lim _{q \rightarrow \infty}\|f\|_{\mathbf{M}^{p q}}=\sup _{y>0} y\left[P\left(f^{*}>y\right)\right]^{\frac{1}{p}}, \quad 0<p<\infty .
$$

Proposition 2.6 ([15]). The map $f \mapsto\|f\|_{\mathbf{M}^{p q}}$ is a quasi-norm; namely, for any two families of functions $f=\left(f_{t}, t \in \mathbf{T}\right)$ and $g=\left(g_{t}, t \in \mathbf{T}\right)$ and for any $\lambda \in C$,

$$
\|\lambda f\|_{\mathbf{M}^{p q}}=|\lambda|\|f\|_{\mathbf{M}^{p q}}, \quad\|f+g\|_{\mathbf{M}^{p q}} \leq K_{p q}\left(\|f\|_{\mathbf{M}^{p q}}+\|g\|_{\mathbf{M}^{p q}}\right),
$$

where $0<p<\infty, 0<q \leq \infty$ and $K_{p q}$ depends only on $p$ and $q$. Moreover, the map $f \mapsto\|f\|_{\mathbf{M}^{p q}}$ is non-decreasing in the following sense: If for all $t \in \mathbf{T}$ the inequality $\left\|f_{t}\right\| \leq\left\|g_{t}\right\|$ holds, then

$$
\|f\|_{\mathbf{M}^{p q}} \leq\|g\|_{\mathbf{M}^{p q}} \quad(0<p<\infty, 0<q \leq \infty) .
$$

Remark 2.7. Note that if $\mathbf{T}$ is linearly ordered, then the sets $\left\{t \in \nu_{y}^{f}\right\}(t \in \mathbf{T})$ are pairwise disjoint and $\sum_{t \in \mathbf{T}} \chi_{\left\{t \in \nu_{y}^{f}\right\}}=\chi_{\left\{f^{*}>y\right\}}$. In this case,

$$
\|f\|_{\mathbf{M}^{p q}}=\sup _{y>0} y\left[P\left(f^{*}>y\right)\right]^{\frac{1}{p}}, \quad 0<p<\infty, 0<q \leq \infty .
$$

The $\mathbf{X}$-valued predictable tree martingale can be defined as a one-parameter predictable martingale. In Definition 2.8 we shall give its concept.

Definition 2.8. We say that an $\mathbf{X}$-valued tree martingale $f=\left(f_{t}, t \in \mathbf{T}\right)$ is predictable if there exists a family $\lambda=\left(\lambda_{t}, t \in \mathbf{T}\right)$ of non-decreasing, non-negative and predictable functions such that

$$
\left\|f_{t}\right\| \leq \lambda_{t} \quad(t \in \mathbf{T})
$$

where non-decreasing functions means that for any comparable elements $s, t \in \mathbf{T}$, if $s \leq t$, then $\lambda_{s} \leq \lambda_{t}$; predictable functions means that for any $t \in \mathbf{T}, \lambda_{t}$ is $\mathcal{F}_{t}^{-}$-measurable. Such a $\lambda$ is called a prediction belonging to $f$.

Denote by $\mathbf{P}^{p q}$ the space of this kind of $\mathbf{X}$-valued tree martingales and endow it with the following quasi-norm:

$$
\|f\|_{\mathbf{P}^{p q}}=\inf \|\lambda\|_{\mathbf{M}^{p q}} \quad(0<p<\infty, 0<q \leq \infty)
$$

where the infimum is taken over all predictions $\lambda \in \mathbf{M}^{p q}$ belonging to $f$. For the reader's convenience, throughout this paper, we always let

$$
\left\|\left(\pi_{t} f, t \in \mathbf{T}\right)\right\|_{\mathbf{M}^{p q}}=\|\pi f\|_{\mathbf{M}^{p q}}, \quad\left\|\left(f_{t}, t \in \mathbf{T}\right)\right\|_{\mathbf{M}^{p q}}=\|f\|_{\mathbf{M}^{p q}} .
$$




\section{SOME QUASI-NORMAL INEQUALITIES FOR VECTOR-VALUED TREE MARTINGALE TRANSFORMS IN UMD SPACES}

In this section, we shall prove some quasi-normal inequalities for vector-valued tree martingale transforms in a $U M D$ space $\mathbf{X}$, and these inequalities shall be used in the next section.

Theorem 3.1. Assume that $\mathbf{X}$ is a $U M D$ space and that an $\mathbf{X}$-valued tree martingale $f=\left(f_{t}, t \in \mathbf{T}\right)$ is predictable. If $1<p \leq q<\infty$, then there exists a constant $C_{p, q}$ depending only on $p$ and $q$ such that

$$
\left\|\left(\pi_{t} f, t \in \mathbf{T}\right)\right\|_{\mathbf{M}^{p \infty}} \leq C_{p, q}\|f\|_{\mathbf{P}^{p q}} .
$$

We need to introduce some knowledge of a $U M D$ space $\mathbf{X}$. Since for every $t \in \mathbf{T}$, $\mathbf{T}_{t}$ is a totally ordered set, we see that $f=\left(f_{t}, t \in \mathbf{T}_{t}\right)$ is a sequence of $\mathbf{X}$-valued linearly ordered martingales, where $f_{t_{0}}=0, \mathcal{F}_{t_{0}}=\{\emptyset, \Omega\}$. Let $v=\left(v_{t}, t \in \mathbf{T}\right)$ be a family of real valued predictable sequences. Then for every $t \in \mathbf{T}, v=\left(v_{t}, t \in \mathbf{T}_{t}\right)$ is a linearly ordered predictable sequence. Therefore, the transforms of the martingale sequence $f=\left(f_{t}, t \in \mathbf{T}_{t}\right)$ can be defined by

$$
g_{s}=\sum_{t_{0} \leq r<s} v_{r}\left(d_{r} f\right)\left(f_{t_{0}}=0, t_{0}, r, s \in \mathbf{T}_{t}\right), \quad g=\left(g_{s}, s \in \mathbf{T}_{t}\right) .
$$

It follows from [3] that if $E\left\|g_{s}\right\|<\infty\left(s \in \mathbf{T}_{t}\right)$, then $g$ is a martingale.

Definition 3.2 ([4]). If for any $\mathbf{X}$-valued martingale $f=\left(f_{t}, t \in \mathbf{T}_{t}\right)$ and its martingale difference sequences $\left(d_{u} f, u \in \mathbf{T}_{t}\right), \mathbf{T}_{t}$ is a totally ordered set, it holds that

$$
\left\|\sum_{t_{0} \leq r<s} v_{r}\left(d_{r} f\right)\right\|_{p} \leq C_{p}\left\|\sum_{t_{0} \leq r<s}\left(d_{r} f\right)\right\|_{p},
$$

where $v_{r}=+1$ or $v_{r}=-1$ and $1<p<\infty$. Then the space $\mathbf{X}$ is called a space in which martingale difference sequences are unconditional. That is, the space $\mathbf{X} \in U M D$.

The relationship between the space $\mathbf{X} \in U M D$ and $\mathbf{X}$-valued martingale transforms is given in the following lemma.

Lemma 3.3. The space $\mathbf{X} \in U M D$ if and only if for any $\mathbf{X}$-valued martingale $f=\left(f_{s}, s \in \mathbf{T}_{t}\right), \mathbf{T}_{t}$ is a totally ordered set and, for $1<p<\infty$, it holds that

$$
\left\|g_{s}\right\|_{p} \leq C_{p}\left\|f_{s}\right\|_{p} \quad\left(s \in \mathbf{T}_{t}\right)
$$

where $g_{s}\left(s \in \mathbf{T}_{t}\right)$ is the transform of an $\mathbf{X}$-valued martingale $f$.

Proof. Since $\mathbf{T}_{t}$ is a totally ordered set and linearly ordered, we can introduce a $\operatorname{map} \varphi: \mathbf{T}_{t} \rightarrow \mathbf{N} \cup\{0\}$, where $\mathbf{N}$ is a nonnegative integer set. Therefore, we can construct a map $\varphi$ such that $f=\left(f_{t}, t \in \mathbf{T}_{t}\right) \rightarrow \varphi(f)=\left(f_{n}, n \in \mathbf{N} \cup\{0\}\right)$ and $\varphi$ is a one-to-one map. So the inequality (3.4) follows from Burkholder's result [4] that for any one-parameter martingale $f=\left(f_{n}, n \in \mathbf{N} \cup\{0\}\right)$, the inequality $\left\|g_{n}\right\|_{p} \leq C_{p}\left\|f_{n}\right\|_{p}(n \in \mathbf{N} \cup\{0\})$ holds.

Lemma 3.4 ([15]). Let $\mathbf{T}$ be a countable index set and $\left(\mathcal{F}_{t}, t \in \mathbf{T}\right)$ be an arbitrary (not necessarily monotone) family of $\sigma$-algebras with the assumption $\sigma\left(\bigcup_{t \in \mathbf{T}} \mathcal{F}_{t}\right)=$ $\mathcal{F}$. Suppose that for all $h \in L_{p}$ that Doob's inequality

$$
\left\|\sup _{t \in \mathbf{T}}\left|E_{t} h\right|\right\|_{p} \leq C_{p}\|h\|_{p} \quad(p>1)
$$


holds. If $f=\left(f_{t}, t \in \mathbf{T}\right)$ is a family of non-negative measurable functions, then for $1 \leq p<\infty$, we have

$$
\begin{aligned}
& E\left(\sum_{t \in \mathbf{T}} E_{t} f_{t}\right)^{p} \leq C_{q} E\left(\sum_{t \in \mathbf{T}} f_{t}\right)^{p}, \\
& E\left(\sum_{t \in \mathbf{T}} f_{t}\right)^{\frac{1}{p}} \leq B_{p} E\left(\sum_{t \in \mathbf{T}} E_{t} f_{t}\right)^{\frac{1}{p}},
\end{aligned}
$$

where $1 / p+1 / q=1$, and $B_{p}>0$ and $C_{q}>0$ denote the constant in (3.5).

For the proof of Theorem 3.1, we need some additional lemmas, which we shall prove.

Lemma 3.5. Assume that an $\mathbf{X}$-valued tree martingale $f=\left(f_{t}, t \in \mathbf{T}\right)$ is predictable and $\lambda=\left(\lambda_{t}, t \in \mathbf{T}\right)$ is a prediction belonging to $f$. Then for any real number $x>0$ set $\varepsilon_{t}=\chi_{\left\{x<\lambda_{t^{+}} \leq 2 x\right\}}(t \in \mathbf{T})$ and $\varepsilon=\left(\varepsilon_{t}, t \in \mathbf{T}\right)$. It holds that

$$
\pi_{\varepsilon} f \leq 2 \sup _{t \in \mathbf{T}} \alpha_{t} \pi_{\varepsilon, t}(f)+4 R x \chi_{\left\{\lambda^{*}>x\right\}}
$$

where $\alpha_{t}=\chi_{\left\{\lambda_{t}>y, \lambda_{s} \leq y, s<t\right\}}(s, t \in \mathbf{T})$ and $\lambda^{*}=\sup _{t \in \mathbf{T}} \lambda_{t}$ is the maximal function of $\lambda$.

Proof. Let us fix $t$ and $t<s(t, s \in \mathbf{T})$, and set

$$
\tau_{r}=\chi_{\left\{\lambda_{r}>x\right\}}, \quad r \in \mathbf{T}, \omega \in \Omega .
$$

It is clear that $\tau_{r^{+}}=0$ implies $\varepsilon_{r}=0$ and hence $\varepsilon_{r}=\varepsilon_{r} \tau_{r^{+}}$. Therefore, if the set

$$
\left\{r \in \mathbf{T}: t \leq r<s, \tau_{r^{+}}(\omega)=1\right\}
$$

is empty, then $\pi_{\varepsilon, t, s} f(\omega)=\sum_{t \leq r<s}\left\|\varepsilon_{r} \pi^{r}\left(d_{r} f(\omega)\right)\right\|=0$. Otherwise, let $t_{1}$ be its minimum element and denote by $t_{0}$ a minimum element of the set $\{r \in \mathbf{T}: r \leq$ $\left.t_{1}^{+}, \tau_{r}(\omega)=1\right\}$; we see that $\alpha_{t_{0}}=1$. Consequently, and by using the definition of $\varepsilon_{t}$, we can derive that

$$
\begin{aligned}
\pi_{\varepsilon, t, s} f(\omega) & =\pi_{\varepsilon, t_{1}, s} f(\omega)=\varepsilon_{t_{1}} \pi^{t_{1}}\left(d_{t_{1}} f\right)(w)+\pi_{\varepsilon, t_{1}^{+}, s} f(\omega) \\
& =\pi^{t_{1}}\left(\varepsilon_{t_{1}} d_{t_{1}} f\right)(\omega)+\alpha_{t_{0}}(\omega)\left[\pi_{\varepsilon, t_{0}, s} f(\omega)-\pi_{\varepsilon, t_{0}, t_{1}^{+}} f(\omega)\right] .
\end{aligned}
$$

It follows from the predictability of the $\mathbf{X}$-valued tree martingale $f$ and (4) of Definition 2.3 that

$$
\begin{aligned}
\left\|\pi^{t_{1}}\left(\varepsilon_{t_{1}} d_{t_{1}} f\right)\right\| & \leq R\left[E_{t_{1}}\left(\varepsilon_{t_{1}}\left\|d_{t_{1}} f\right\|\right)\right] \\
& \leq R\left[E_{t_{1}}\left(4 \varepsilon_{t_{1}} \lambda_{t_{1}^{+}}\right)\right] \leq 4 R x \chi_{\left\{\lambda^{*}>x\right\}} .
\end{aligned}
$$

On the other hand,

$$
\left\|\pi_{\varepsilon, t_{0}, s} f(\omega)-\pi_{\varepsilon, t_{0}, t_{1}^{+}} f(\omega)\right\| \leq 2 \pi_{\varepsilon, t_{0}} f(w) .
$$

Substituting (3.10) and (3.11) into (3.9) yields that

$$
\pi_{\varepsilon, t, s} f(\omega) \leq 2 \alpha_{t} \pi_{\varepsilon, t}(f)+4 R x \chi_{\left\{\lambda^{*}>x\right\}} .
$$

Taking the supremum over all $s \in \mathbf{T}_{t}$ and $t \in \mathbf{T}$, we get (3.8). The proof is complete. 
Lemma 3.6. Let $f=\left(f_{t}, t \in \mathbf{T}\right), \lambda=\left(\lambda_{t}, t \in \mathbf{T}\right), \varepsilon=\left(\varepsilon_{t}, t \in \mathbf{T}\right)$ and $\left(\alpha_{t}, t \in \mathbf{T}\right)$ be as in Lemma 3.5. If the space $\mathbf{X} \in U M D$ and $1<p \leq q<\infty$, then for any real numbers $x, z>0$, there exists a constant $C_{p, q}$ depending only on $p$ and $q$ such that

$$
P(Y>z x) \leq C_{p, q} z^{-q} x^{-p}\|\lambda\|_{\mathbf{M}^{p q}}^{p}
$$

where $Y$ denotes the operator $\sup _{t \in \mathbf{T}} \alpha_{t} \pi_{\varepsilon, t} f$.

Proof. Suppose $Y=\sup _{t \in \mathbf{T}} \alpha_{t} \pi_{\varepsilon, t} f$. It follows from (3) of Definition 2.3 that for any non-negative $\mathcal{F}_{t}$-measurable function $\xi$,

$$
\pi_{\varepsilon, t}(\xi f)=\xi \pi_{\varepsilon, t} f .
$$

By (1) of Definition 2.1 and (1) of Definition 2.3 we can see that

$$
\pi^{r}\left(d_{r} f\right)=P_{r^{+}}\left(\pi^{r}\left(d_{r} f\right)\right)=\phi_{t} E_{t^{+}}\left[\pi^{r}\left(d_{r} f\right) \bar{\phi}_{t}\right] \quad(r \geq t)
$$

hence $\bar{\phi}_{t} \pi^{r}\left(d_{r} f\right)$ is $\mathcal{F}_{r^{+}}$-measurable. On the other hand, it follows from (2) of Definition 2.3 that

$$
P_{r} \pi^{r}\left(d_{r} f\right)=\phi_{t} E_{r}\left[\pi^{r}\left(d_{r} f\right) \bar{\phi}_{t}\right]=0 \quad(r \geq t) ;
$$

therefore, $\left(\bar{\phi}_{t} \pi^{r}\left(d_{r} f\right)\right)_{r \geq t}$ is a one-parameter martingale difference sequence relative to $\left(\mathcal{F}_{r^{+}}\right)_{r \geq t}$. So the one-parameter martingale inequalities can be applied here. Since $\left|\phi_{t}\right|=1$, we have for every $t \in \mathbf{T}$,

$$
\pi_{\varepsilon, t} f=\sup _{s \geq t}\left\|\sum_{t \leq r<s} \varepsilon_{r} \pi^{r}\left(d_{r} f\right) \bar{\phi}_{t}\right\| .
$$

Using (4) of Definition 2.3 and Lemma 3.3 together with the convexity theorem (Lemma 3.4) and (3.13) we obtain that for $p_{0}>1$,

$$
\begin{aligned}
E_{t}\left[\left(\pi_{\varepsilon, t} f\right)^{p_{0}}\right] & =E_{t}\left[\left(\sup _{s \geq t}\left\|\sum_{t \leq r<s} \varepsilon_{r} \pi^{r}\left(d_{r} f\right) \bar{\phi}_{t}\right\|\right)^{p_{0}}\right] \leq E_{t}\left[\left(\sup _{s \geq t} \sum_{t \leq r<s} \varepsilon_{r}\left\|\pi^{r}\left(d_{r} f\right) \bar{\phi}_{t}\right\|\right)^{p_{0}}\right] \\
& \leq R^{p_{0}} E_{t}\left[\left(\sup _{s \geq t} \sum_{t \leq r<s} \varepsilon_{r} E_{r}\left\|\left(d_{r} f\right)\right\|\right)^{p_{0}}\right] \quad\left(\text { by }\left|\phi_{t}\right|=1\right. \text { and (4) of Def. 2.3) } \\
& \leq C_{p_{0}} E_{t}\left[\left(\sup _{s \geq t} \sum_{t \leq r<s}\left\|\varepsilon_{r}\left(d_{r} f\right)\right\|\right)^{p_{0}}\right] \quad \text { (by Convexity Theorem) } \\
& \leq C_{p_{0}} E_{t}\left[\sup _{s \geq t} \varepsilon_{s}\left\|f_{s}\right\|^{p_{0}}\right] .
\end{aligned}
$$

It follows from the definition of $\varepsilon_{s}(s \in \mathbf{T})$ and the previous inequality that

$$
E_{t}\left[\left(\pi_{\varepsilon, t} f\right)^{p_{0}}\right] \leq C_{p_{0}} x^{p_{0}} .
$$

For $p_{0} \geq q \geq 1$, we see easily that $0<\frac{q}{p_{0}} \leq 1$. By using Tsebisev's inequality and the concavity theorem (Lemma 3.4) and (3.17) we can derive that

$$
\begin{aligned}
P(Y>z x) & \leq(z x)^{-q} E\left[\left(\sup _{t \in \mathbf{T}} \alpha_{t} \pi_{\varepsilon, t} f\right)^{q}\right] \quad \text { (by Tsebisev's inequality) } \\
& \leq(z x)^{-q} E\left[\left(\sum_{t \in \mathbf{T}} \alpha_{t}\left(\pi_{\varepsilon, t} f\right)^{p_{0}}\right)^{q / p_{0}}\right] \\
& \leq C_{q}(z x)^{-q} E\left[\left(\sum_{t \in \mathbf{T}} \alpha_{t} E_{t}\left[\left(\pi_{\varepsilon, t} f\right)^{p_{0}}\right]\right)^{q / p_{0}}\right] \quad \text { (by Concavity Theorem) } \\
& \leq C_{q} z^{-q} E\left[\left(\sum_{t \in \mathbf{T}} \alpha_{t}\right)^{q / p_{0}}\right] \quad(\text { by }(3.17)) .
\end{aligned}
$$


On the other hand, set $p_{0}=q^{2} / p$, since $1 \leq p \leq q<\infty$, so $p_{0} \geq q>1$. Then using the definition of quasi-norm $\|\cdot\|_{M^{p q}}$ we can derive that

$$
E\left[\left(\sum_{t \in \mathbf{T}} \alpha_{t}\right)^{q / p_{0}}\right]=E\left[\left(\sum_{t \in \mathbf{T}} \chi_{\left\{\lambda_{t}>x, \lambda_{s} \leq x, s<t\right\}}\right)^{p / q}\right] \leq x^{-p}\|\lambda\|_{\mathbf{M}^{p q}}^{p} .
$$

Substituting (3.19) into (3.18) yields (3.12). The proof is complete.

Proof of Theorem 3.1. The proof of Theorem 3.1 is analogous to Theorem 2.2 of [9] (73-74), and its proof is also analogous to Theorem 4.7 of [15] (refer to [15] $(150-151))$.

We obtain easily the following corollary from Theorem 3.1.

Corollary 3.7. Assume that $\mathbf{X}$ is a $U M D$ space and that an $\mathbf{X}$-valued oneparameter martingale $f=\left(f_{n}, n \in \mathbf{N}\right)$ is predictable. If $1<p \leq q<\infty$, then there exists a constant $C_{p, q}$ depending only on $p$ and $q$ such that

$$
\sup _{y>0} y[P(\pi f>y)]^{\frac{1}{p}} \leq C_{p, q}\|f\|_{\mathbf{P}^{p q}} .
$$

\section{Maximal operator Inequality For Vector-VAlued TREE MARTINGALE TRANSFORMS}

We need some knowledge of interpolation. Firstly, we introduce some definitions. The distribution function $m(y, f)$ of a vector-valued measurable function $f$ is defined by

$$
m(y, f)=P(\{x:\|f(x)\|>y\}) \quad(y \geq 0)
$$

clearly, $m(y, f)$ is non-increasing and continuous on the right. It is well known that

$$
\|f\|_{p}=\left(p \int_{0}^{\infty} y^{p-1} m(y, f) d y\right)^{\frac{1}{p}}(0<p<\infty),\|f\|_{\infty}=\inf \{y: m(y, f)=0\} .
$$

Using the distribution function $m(y, f)$ we introduce the weak $L_{p}$ spaces denoted by $L_{p}^{*}$. The space $L_{p}^{*}$ consists of all measurable vector-valued functions $f$ satisfying

$$
\|f\|_{L_{p}^{*}}=\sup _{y>0} y m(y, f)^{\frac{1}{p}}<\infty(0<p<\infty),
$$

while we set $L_{\infty}^{*}=L_{\infty}$. Note that $L_{p}^{*}$ is a quasi-normal space; it is easy to see that $L_{p} \subset L_{p}^{*}$ for every $0<p \leq \infty$. In their definition, another concept shall be used. Let $f$ be a vector-valued measurable function. The non-increasing rearrangement of $f$ is defined by

$$
f^{*}(t)=\inf \{y: m(y, f) \leq t\} .
$$

Remark 4.1 ([15]). It is well known that $f^{*}$ is non-increasing, continuous on the right and

$$
m\left(x, f^{*}\right)=m(x, f) \quad(x \geq 0) .
$$

The Lorentz spaces $L_{p q}$ are defined as follows: For $0<p, q<\infty$, define

$$
\begin{gathered}
\|f\|_{p q}=\left(\int_{0}^{\infty} f^{*}(t)^{q} t^{\frac{q}{p}} \frac{d t}{t}\right)^{\frac{1}{q}},\|f\|_{p \infty}=\sup _{t>0} t^{\frac{1}{p}} f^{*}(t), \\
L_{p q}=\left\{f:\|f\|_{p q}<\infty\right\}, \text { and } L_{p \infty}=\left\{f:\|f\|_{p \infty}<\infty\right\} .
\end{gathered}
$$

The spaces $L_{p}^{*}$ are special cases of the more general Lorentz space $L_{p q}$. We have one lemma on Lorentz spaces, here. 
Lemma 4.2 ([15]). Let

$$
L_{p q}=L_{p q}(\Omega, \mathcal{A}, P)=\left\{f:\|f\|_{p q}<\infty\right\} .
$$

Then

$$
L_{p p}=L_{p}, \quad L_{p \infty}=L_{p}^{*} \quad(0<p \leq \infty) .
$$

Assume that $A_{0}$ and $A_{1}$ are quasi-norm spaces embodied continuously in a topological vector space $A$. In the real method of interpolation, the interpolation spaces between $A_{0}$ and $A_{1}$ are defined by means of an interpolating function $K\left(t, f, A_{0}, A_{1}\right)$. If $f \in A_{0}+A_{1}$, define

$$
K\left(t, f, A_{0}, A_{1}\right):=\inf _{f=f_{0}+f_{1}}\left\{\left\|f_{0}\right\|_{A_{0}}+t\left\|f_{1}\right\|_{A_{1}}\right\},
$$

where the infimum is taken over all choices of $f_{0}$ and $f_{1}$ such that $f_{0} \in A_{0}, f_{1} \in A_{1}$ and $f=f_{0}+f_{1}$. The interpolation space $\left(A_{0}, A_{1}\right)_{\theta q}$ is defined by the space of all functions $f$ in $A_{0}+A_{1}$ such that

$$
\|f\|_{\left(A_{0}, A_{1}\right)_{\theta q}}=\left(\int_{0}^{\infty}\left[t^{-\theta} K\left(t, f, A_{0}, A_{1}\right)\right]^{q} \frac{d t}{t}\right)^{\frac{1}{q}}<\infty,
$$

where $0<\theta<1$ and $0<q \leq \infty$. Let $B_{0}$ and $B_{1}$ also be quasi-norm spaces embodied continuously in a topological vector space $B$. Then a map

$$
T: A_{0}+A_{1} \rightarrow B_{0}+B_{1}
$$

is said to be quasi-linear from $\left(A_{0}, A_{1}\right)$ to $\left(B_{0}, B_{1}\right)$ if for given $a \in A_{0}+A_{1}$ and $a_{i} \in A_{i}(i=0,1)$ with $a_{0}+a_{1}=a$, there exist $b_{i} \in B_{i}(i=0,1)$ satisfying

$$
T a=b_{0}+b_{1}
$$

and

$$
\left\|b_{i}\right\|_{B_{i}} \leq K_{i}\left\|a_{i}\right\|_{A_{i}} \quad\left(K_{i}>0, i=0,1\right) .
$$

The following lemma shows that the boundedness of a quasi-linear operator is hereditary for the interpolation spaces.

Lemma 4.3 ([15]). If $0<q \leq \infty, 0 \leq \theta \leq 1$ and $T$ is a quasi-linear map from $\left(A_{0}, A_{1}\right)$ to $\left(B_{0}, B_{1}\right)$, then

$$
T:\left(A_{0}, A_{1}\right)_{\theta q} \rightarrow\left(B_{0}, B_{1}\right)_{\theta q}
$$

and

$$
\|T a\|_{\left(B_{0}, B_{1}\right)_{\theta q}} \leq K_{0}^{1-\theta} K_{1}^{\theta}\|a\|_{\left(A_{0}, A_{1}\right)_{\theta q}} .
$$

Lemma 4.4 ([15]). If $0<p<\infty, 0<\theta<1$, and $p \leq q \leq \infty$, then

$$
\left(L_{p}, L_{\infty}\right)_{\theta q}=L_{r q} \quad\left(\frac{1}{r}=\frac{1-\theta}{p}\right) .
$$

Lemma 4.5 ([1]). Let $T$ be a linear operator which maps sequences of functions to sequences of functions. Suppose that for arbitrary $1 \leq p_{i}, q_{i}, r_{i}, s_{i} \leq \infty(i=0,1), T$ is a bounded operator from $L_{p_{0}}\left(l_{q_{0}}\right)$ to $L_{r_{0}}\left(l_{s_{0}}\right)$ and from $L_{p_{1}}\left(l_{q_{1}}\right)$ to $L_{r_{1}}\left(l_{s_{1}}\right)$. Then $T$ is also bounded from $L_{p}\left(l_{q}\right)$ to $L_{r}\left(l_{s}\right)$ if $p, q<\infty, 0<t<1$,

$$
\frac{1}{p}=\frac{1-t}{p_{0}}+\frac{t}{p_{1}}, \quad \frac{1}{q}=\frac{1-t}{q_{0}}+\frac{t}{q_{1}}
$$

and

$$
\frac{1}{r}=\frac{1-t}{r_{0}}+\frac{t}{r_{1}}, \quad \frac{1}{s}=\frac{1-t}{s_{0}}+\frac{t}{s_{1}} .
$$


Next, we shall introduce the concepts of regular tree martingales and previsible tree martingales.

Definition 4.6. A tree stochastic basis $\mathcal{F}$ is said to be regular if there exists a constant $R>0$ such that for all $f \in L_{1}(\mathbf{X})$,

$$
\left\|E_{t} f\right\| \leq R E_{t}^{-}\left\|E_{t} f\right\| \quad(t \in \mathbf{T}),
$$

where $E_{t}^{-}$denotes the conditional expectation operator with respect to $\mathcal{F}_{t}^{-}$.

Definition 4.7. We say that a family of integrable functions $f=\left(f_{t}, t \in \mathbf{T}\right)$ is a vector-valued regular tree martingale if $s \leq t$ implies $P_{s} f_{t}=f_{s}$ and there exists a constant $R>0$ such that

$$
\left\|f_{t}\right\| \leq R\left\|P_{s} f_{t}\right\| \quad(t \in \mathbf{T}) .
$$

Definition 4.8. A vector-valued tree martingale $f=\left(f_{t}, t \in \mathbf{T}\right)$ is previsible if there exists an $N \in \mathbf{N}$ such that for every $t \in \mathbf{T}$ there are indices $t_{1}, \ldots, t_{N} \in \mathbf{T}$ satisfying $t_{i}^{+}=t(i=1, \ldots, N)$ and

$$
\left\|f_{t}\right\| \leq C \sum_{i=1}^{N}\left\|f_{t_{i}}\right\| \quad(t \in \mathbf{T}) .
$$

The following theorem is important to verify maximal operator inequality of $\mathbf{X}$-valued tree martingale transforms.

Theorem 4.9. Let $1<p<\infty$. Assume that $\mathbf{X}$ is a $U M D$ space and that an $\mathbf{X}$-valued tree martingale $f=\left(f_{t}, t \in \mathbf{T}\right) \in L_{p}(\mathbf{X})$.

(i) If $f=\left(f_{t}, t \in \mathbf{T}\right)$ is previsible, then there exists a constant $C_{p}$ such that

$$
\left\|\left(\pi_{t} f, t \in \mathbf{T}\right)\right\|_{\mathbf{M}^{p \infty}} \leq C_{p}\|f\|_{p} .
$$

(ii) If $f=\left(f_{t}, t \in \mathbf{T}\right)$ is regular, then the inequality (4.13) also holds.

To prove Theorem 4.9, some necessary lemmas shall be shown in part. Let $\tau$ : $\mathbf{T} \longrightarrow \mathbf{T}$ be a map such that $\tau(t) \leq t$ and $\mathcal{F}_{\tau(u)} \subset \mathcal{F}_{\tau(t)}$ for any comparable $u \leq t$ $(u, t \in \mathbf{T})$. Here, we set

$$
f_{t}^{\tau}=E_{\tau(t)}\left\|f_{t}\right\|, \quad f^{\tau}=\left(f_{t}^{\tau}, t \in \mathbf{T}\right) .
$$

Lemma 4.10. Let $f=\left(f_{t}, t \in \mathbf{T}\right)$ be an $\mathbf{X}$-valued tree martingale. If $1<p, q<\infty$ and satisfy

$$
\frac{1}{p}=\frac{1-t}{2}+\frac{t}{s}, \quad \frac{1}{q}=\frac{1-t}{2} \quad(0 \leq t \leq 1,1<s \leq \infty),
$$

then for all $f \in L_{p}(\mathbf{X})$, there exists a constant $C_{p, q}$ depending only on $p$ and $q$ such that

$$
\left\|\left(f_{t}^{\tau}, t \in \mathbf{T}\right)\right\|_{\mathbf{M}^{p q}} \leq C_{p, q}\|f\|_{p} .
$$

Proof. For a fixed $y>0$ and $f \in L_{p}(\mathbf{X})$, let

$$
\alpha_{t}=\chi_{\left\{t \in v_{y}^{f^{\tau}}\right\}}=\chi_{\left\{f_{t}^{\tau}>y, f_{u}^{\tau} \leq y, u<t\right\}} \quad(u, t \in \mathbf{T}) .
$$

Then $\alpha_{t}$ is $\mathcal{F}_{\tau(t)}$-measurable, and obviously,

$$
\alpha_{t} \alpha_{u}=0 \text { as } t<u \text { or } u<t \quad(u, t \in \mathbf{T}) .
$$


For all $t \in \mathbf{T}$, we shall introduce a family of projections $H_{t}:=\alpha_{t} P_{t}(t \in \mathbf{T})$, and by the martingale property, we observe that

$$
P_{t} \circ P_{u}=0 \text {, as } t \text { and } u \text { are incomparable. }
$$

Therefore, we can derive that for any $g \in L_{1}(\mathbf{X})$ and $t, u \in \mathbf{T}$,

$$
H_{t}\left(H_{u} g\right)=\alpha_{t} P_{t}\left(P_{u}\left(\alpha_{u} g\right)\right)=P_{t}\left(\alpha_{t} \alpha_{u} P_{u} g\right)=\delta_{t, u} H_{t} g,
$$

where $\delta_{t, u}$ is the Kronecker symbol. Thus the family of projections $H_{t}(t \in \mathbf{T})$ are orthogonal. For any family of complex numbers $c=\left(c_{t}, t \in \mathbf{T}\right)$ and $g \in L_{2}(\mathbf{X})$, using the orthogonality of $H g=\left(H_{t} g, t \in \mathbf{T}\right)$ we can derive that

$$
\begin{aligned}
\left\|g-\sum_{t \in \mathbf{T}} c_{t} H_{t} g\right\|_{L_{2}\left(l_{2}\right)}^{2} & =\left(g-\sum_{t \in \mathbf{T}} c_{t} H_{t} g, g-\sum_{t \in \mathbf{T}} c_{t} H_{t} g\right) \\
& =\|g\|_{2}^{2}-\sum_{t \in \mathbf{T}} c_{t} \overline{H_{t} g}-\sum_{t \in \mathbf{T}} \overline{c_{t}} H_{t} g+\sum_{t \in \mathbf{T}}\left\|c_{t}\right\|_{2}^{2} \\
& =\|g\|_{2}^{2}-\sum_{t \in \mathbf{T}}\left\|H_{t} g\right\|_{2}^{2}+\sum_{t \in \mathbf{T}}\left\|H_{t} g-c_{t}\right\|_{2}^{2} .
\end{aligned}
$$

Let $c_{t}=H_{t} g \quad(t \in \mathbf{T})$. Then substituting it into (4.16) we obtain that

$$
\left\|g-\sum_{t \in \mathbf{T}} c_{t} H_{t} g\right\|_{L^{2}\left(l^{2}\right)}^{2}=\|g\|_{2}^{2}-\sum_{t \in \mathbf{T}}\left\|H_{t} g\right\|_{2}^{2},
$$

and by (4.17) we can further derive that

$$
\left\|\left(H_{t} g, t \in \mathbf{T}\right)\right\|_{L_{2}\left(l_{2}\right)}^{2}=\sum_{t \in \mathbf{T}}\left\|H_{t} g\right\|_{2}^{2} \leq\|g\|_{2}^{2} .
$$

To prove (4.14) we introduce a family of operators

$$
Q_{t} g:=E_{\tau(t)}\left(\eta_{t} H_{t} g\right) \quad\left(g \in L_{1}, t \in \mathbf{T}\right),
$$

where $\left(\eta_{t}, t \in \mathbf{T}\right)$ is a fixed family of functions satisfying $\left\|\eta_{t}\right\|_{\infty} \leq 1$ for any $t \in \mathbf{T}$. Using (4.18) one sees that

$$
\begin{aligned}
\left\|\left(Q_{t} g, t \in \mathbf{T}\right)\right\|_{L_{2}\left(l_{2}\right)}^{2} & \leq E\left(\sum_{t \in \mathbf{T}} E_{\tau(t)}\left\|H_{t} g\right\|^{2}\right) \\
& =E\left(\sum_{t \in \mathbf{T}}\left\|H_{t} g\right\|^{2}\right) \leq\|g\|_{2}^{2} .
\end{aligned}
$$

On the other hand, by Doob's inequality, it follows that for any $1<s \leq \infty$ and $g \in L_{s}$

$$
\left\|\left(Q_{t} g, t \in \mathbf{T}\right)\right\|_{L_{s}\left(l_{\infty}\right)} \leq\left\|\sup _{t \in \mathbf{T}} E_{\tau(t)}\right\| g\|\|_{s} \leq \frac{s}{1-s}\|g\|_{s}
$$

By the assumptions: $\frac{1}{p}=\frac{1-t}{2}+\frac{t}{s}$ and $\frac{1}{q}=\frac{1-t}{2}(t \in[0,1])$ and (4.20), (4.21), we see that the assumptions of Lemma 4.5 hold; therefore, applying Lemma 4.5 to the linear operators $\left(Q_{t} g, t \in \mathbf{T}\right)$ we obtain that

$$
\left\|\left(Q_{t} g, t \in \mathbf{T}\right)\right\|_{L_{p}\left(l_{q}\right)} \leq C_{p, q}\|g\|_{p} \quad\left(g \in L_{p}(\mathbf{X})\right) .
$$

Setting $g=f$ and $\eta_{t}=\operatorname{sign} P_{t} f(t \in \mathbf{T})$ we have

$$
\left(E\left[\sum_{t \in T}\left(\alpha_{t} E_{\tau(t)}\left\|f_{t}\right\|\right)^{q}\right]^{\frac{p}{q}}\right)^{\frac{1}{p}} \leq C_{p, q}\|f\|_{p} .
$$


Using the fact that

$$
\alpha_{t} E_{\tau(t)}\left\|f_{t}\right\|=\alpha_{t} f_{t}^{\tau}>y \alpha_{t}(t \in \mathbf{T}),
$$

we can see that (4.14) holds. The proof is complete.

Lemma 4.11. If the assumptions of Lemma 4.10 hold, then for all $f \in L_{p}(\mathbf{X})$, there exists a constant $C_{p, q}$ depending only on $p$ and $q$ such that

$$
\left\|\left(f_{t}, t \in T\right)\right\|_{\mathbf{M}^{p q}} \leq C_{p, q}\|f\|_{p} .
$$

Proof. Since $\left\|f_{t}\right\|$ is $\mathcal{F}_{t}$-measurable for all $t \in \mathbf{T}$, in the special case $\tau(t)=t$ we derive Lemma 4.11 from the result of Lemma 4.10.

Lemma 4.12. If the assumptions of Lemma 4.10 hold, then for all $f=$ $\left(f_{t}, t \in \mathbf{T}\right) \in L_{p}(\mathbf{X})$, we have the following.

(i) If $f=\left(f_{t}, t \in \mathbf{T}\right)$ is previsible, then there exists a constant $C_{p, q}$ depending only on $p$ and $q$ such that

$$
\|f\|_{\mathbf{P}^{p q}} \leq C_{p, q}\|f\|_{p} .
$$

(ii) If $f=\left(f_{t}, t \in \mathbf{T}\right)$ is regular, then the inequality (4.26) also holds.

Proof. (i) Assume that $f$ is previsible. By the definition of a tree $\mathbf{T}$, we easily see that for every $t \in \mathbf{T}$, the set $\{u \mid u \leq t\}$ is finite, and by (4.12) of Definition 4.8, we see that the family

$$
\lambda_{t}=C \sup _{u \leq t} \sum_{i=1}^{N}\left\|f_{u_{i}}\right\| \quad(t \in \mathbf{T})
$$

is a prediction of $f$; therefore,

$$
\left\|f_{t}\right\| \leq \lambda_{t} \leq C \sum_{i=1}^{N} \sup _{u \leq t}\left\|f_{u_{i}}\right\| \quad(t \in \mathbf{T}) .
$$

Since the quasi-norm $\mathbf{M}^{p q}$ is non-decreasing (Proposition 2.6), using (4.27) we can derive that

$$
\begin{aligned}
\|f\|_{\mathbf{P}^{p q}} & \leq\|\lambda\|_{\mathbf{M}^{p q}} \leq C\left\|\sum_{i=1}^{N} \sup _{u \leq t}\right\| f_{u_{i}}\|\|_{\mathbf{M}^{p q}} \leq C \sum_{i=1}^{N}\left\|\sup _{u \leq t}\right\| f_{u_{i}}\|\|_{\mathbf{M}^{p q}} \\
& =C \sum_{i=1}^{N}\left\|\left(f_{u_{i}}, u_{i} \in \mathbf{T}\right)\right\|_{\mathbf{M}^{p q}} .
\end{aligned}
$$

If $u$ and $t$ are incomparable, then $u_{i}$ and $t_{j}(u, t \in \mathbf{T} ; i, j=1, \ldots, N)$ are also incomparable. By Lemma 4.11 and (4.28) we can further derive that

$$
\|f\|_{\mathbf{P}^{p q}} \leq C N C_{p q}\|f\|_{p} .
$$

(ii) Assume that $f$ is regular. Let $\tau(u):=u_{i}$ for any $1 \leq i \leq N(u \in \mathbf{T})$. By (4.10) of Definition 4.6, we see that $\left\|f_{t}\right\| \leq R E_{t}^{-}\left\|f_{t}\right\|(t \in \mathbf{T})$ and the family $\lambda_{t}=R \sup _{u \leq t} E_{t}^{-}\left\|f_{t}\right\|(t \in \mathbf{T})$ is a prediction of $f$. (ii) The proof of Lemma 4.12 can be derived from Lemma 4.10. The proof is complete.

Proof of Theorem 4.9. Theorem 4.9 comes immediately from Theorem 3.1 and Lemma 4.12.

In Theorem 4.13 we shall give a maximal operator inequality of $\mathbf{X}$-valued tree martingale transforms. 
Theorem 4.13. Let $1<p \leq q<\infty$. Assume that $\mathbf{X}$ is a $U M D$ space and that an $\mathbf{X}$-valued tree martingale $f=\left(f_{t}, t \in \mathbf{T}\right) \in L_{p}(\mathbf{X})$.

(i) If $f=\left(f_{t}, t \in \mathbf{T}\right)$ is previsible, then there exists a constant $C$ such that

$$
\|\pi f\|_{q} \leq C\|f\|_{q} .
$$

(ii) If $f=\left(f_{t}, t \in \mathbf{T}\right)$ is regular, then the inequality (4.29) also holds.

For every $\mathbf{X}$-valued tree martingale $f=\left(f_{t}, t \in \mathbf{T}\right)$, the maximal operator of tree martingale transforms $\pi$ maps $f$ to $\pi f$. We define two kinds of spaces for $\pi f$ in the forms

$$
\begin{aligned}
& \widetilde{H}_{r q}(\mathbf{X})=\left\{\pi f:\|\pi f\|_{\widetilde{H}_{r q}(\mathbf{X})}=\|\pi f\|_{r q}<\infty\right\} \\
& \widetilde{H}_{r \infty}(\mathbf{X})=\left\{\pi f:\|\pi f\|_{\widetilde{H}_{r \infty}(\mathbf{X})}=\|\pi f\|_{r \infty}<\infty\right\} .
\end{aligned}
$$

Lemma 4.14. Let an $\mathbf{X}$-valued tree martingale $f=\left(f_{t}, t \in \mathbf{T}\right) \in L_{p}(\mathbf{X})$. If $0<p<\infty, 0<\theta<1, p \leq q \leq \infty$ and $\frac{1}{r}=\frac{1-\theta}{p}$, then there exists a constant $C_{p q r}$ such that

$$
\|\pi f\|_{r q} \leq C_{p q r}\|\pi f\|_{\left(\widetilde{H}_{p p}(\mathbf{X}), \widetilde{H}_{p \infty}(\mathbf{X})\right)_{\theta q}} .
$$

Proof. We make martingale decompositions $f=f_{0}+f_{1}, f_{0}=\left(f_{0 t}, t \in \mathbf{T}\right), f_{1}=$ $\left(f_{1 t}, t \in \mathbf{T}\right)$ such that $\pi f_{0} \in \widetilde{H}_{p p}(\mathbf{X}), \pi f_{1} \in \widetilde{H}_{p \infty}(\mathbf{X})$ with $\pi f \in \widetilde{H}_{p p}(\mathbf{X})+\widetilde{H}_{p \infty}(\mathbf{X})$. Set

$$
K(t, \pi f)=\inf _{\pi f=\pi f_{0}+\pi f_{1}}\left\{\left\|\pi f_{0}\right\|_{\widetilde{H}_{p p}(\mathbf{X})}+t\left\|\pi f_{1}\right\|_{\widetilde{H}_{p \infty}(\mathbf{X})}\right\} .
$$

Then

$$
\begin{aligned}
\int_{0}^{t^{p}}\left(\pi^{*} f\right)^{p}(\tau) d \tau & \leq \int_{0}^{t^{p}}\left(\pi^{*} f_{0}(\tau)+\left\|\pi f_{1}\right\|_{\infty}\right)^{p} d \tau \\
& \leq C_{p}\left(\left\|\pi f_{0}\right\|_{\widetilde{H}_{p p}(\mathbf{X})}+t\left\|\pi f_{1}\right\|_{\widetilde{H}_{p \infty}(\mathbf{X})}\right)^{p},
\end{aligned}
$$

where $\pi^{*} f$ is the non-increasing rearranged operator of the operator $\pi f$. Taking the infimum over all the decompositions of a tree martingale $f$, it follows from (4.31) and (4.32) that

$$
C_{p}^{-1} \int_{0}^{t^{p}}\left(\pi^{*} f\right)^{p}(\tau) d \tau \leq K(t, \pi f)^{p} .
$$

Using (4.33) we can derive that

$$
\pi^{*} f\left(t^{p}\right) \leq\left(t^{-p} \int_{0}^{t^{p}}\left(\pi^{*} f\right)^{p}(\tau) d \tau\right)^{\frac{1}{p}} \leq C_{p} t^{-1} K(t, \pi f) .
$$

Substituting (4.34) into this we can further derive that for $q<\infty$,

$$
\begin{aligned}
\|\pi f\|_{r q}^{q} & =\frac{q}{r} \int_{0}^{\infty}\left(t^{\frac{1}{r}} \pi^{*} f(t)\right)^{q} \frac{d t}{t} \\
& \leq C_{p q r} \int_{0}^{\infty}\left(t^{\frac{p}{r}-1} K(t, \pi f)\right)^{q} \frac{d t}{t}=C_{p q r}\|\pi f\|_{\left(\widetilde{H}_{p p}(\mathbf{X}), \widetilde{H}_{p \infty}(\mathbf{X})\right)_{\theta q}}^{q} .
\end{aligned}
$$

The proof is complete. 
Proof of Theorem 4.13. Firstly, by the definition of the quasi-norms $\|\cdot\|_{\mathbf{M}^{p \infty}}$ and $\|\cdot\|_{L_{p}^{*}}$ we see that $\|\pi f\|_{\mathbf{M}^{p \infty}}=\|\pi f\|_{L_{p}^{*}}$; then it follows from Lemma 4.2 that

$$
\|\pi f\|_{\mathbf{M}^{p \infty}}=\|\pi f\|_{L_{p}^{*}}=\|\pi f\|_{\widetilde{H}_{p \infty}} .
$$

Using (4.35) and Theorem 4.9, we can derive that

$$
\|\pi f\|_{\widetilde{H}_{p \infty}} \leq C_{p}\|f\|_{p} .
$$

Secondly, we make a martingale decomposition $D: f=f_{0}+f_{1}, f_{0}=\left(f_{0 t}, t \in \mathbf{T}\right), f_{1}=$ $\left(f_{1 t}, t \in \mathbf{T}\right)$. In particular, let $f_{0 t}=0(t \in \mathbf{T})$. Then $f_{t}=f_{0 t}+f_{1 t}=f_{1 t}(t \in \mathbf{T})$, $f=f_{1}$. Therefore, $\pi f=\pi f_{0}+\pi f_{1}$ and $\pi f_{0} \in \widetilde{H}_{p p}(\mathbf{X})$, and it follows from (4.36) that

$$
\left\|\pi f_{1}\right\|_{\widetilde{H}_{p \infty}} \leq C_{p}\left\|f_{1}\right\|_{p}=C_{p}\|f\|_{p} .
$$

By $f \in L_{p}(\mathbf{X})$ and (4.37), one sees that $\pi f_{1} \in \widetilde{H}_{p \infty}(\mathbf{X})$. That is, there exists a martingale decomposition $D$ such that the operator $\pi$ satisfies $\pi f_{0} \in \widetilde{H}_{p p}(\mathbf{X})$, $\pi f_{1} \in \widetilde{H}_{p \infty}(\mathbf{X})$ and $\pi f=\pi f_{0}+\pi f_{1}$. Thirdly, it is well known that $L_{p}$ and $L_{\infty}$ are norm spaces embodied continuously in a topological vector space $B ; \widetilde{H}_{p p}(\mathbf{X})$ and $\widetilde{H}_{p \infty}(\mathbf{X})$ are also quasi-norm spaces embodied continuously in a topological vector space $B$. In a word, the map $\pi: L_{p}+L_{\infty} \rightarrow \widetilde{H}_{p p}+\widetilde{H}_{p \infty}$ is a quasi-linear map from $\left(L_{p}, L_{\infty}\right)$ to $\left(\widetilde{H}_{p p}, \widetilde{H}_{p \infty}\right)$. Therefore, applying Lemma 4.3 to the map $\pi$ we obtain that

$$
\|\pi f\|_{\left(\widetilde{H}_{p p}, \widetilde{H}_{p \infty}\right)_{\theta q}} \leq K_{0}^{1-\theta} K_{1}^{\theta}\|f\|_{\left(L_{p}, L_{\infty}\right)_{\theta q}}
$$

where $0<\theta<1$ and $1<p \leq q \leq \infty$. Setting $\frac{1}{r}=\frac{1-\theta}{p}$, using Lemma 4.4, (4.30) of Lemma 4.14 and (4.38), we can derive that

$$
\|\pi f\|_{r q} \leq C\|f\|_{r q} .
$$

Let $\theta=\frac{q-p}{q}$; then $r=q$. By Lemma 4.2 we see that

$$
\|\pi f\|_{r q}=\|\pi f\|_{q}, \quad\|f\|_{r q}=\|f\|_{q},
$$

and substituting (4.40) into (4.39) yields that

$$
\|\pi f\|_{q} \leq C\|f\|_{q}
$$

The proof is complete.

Theorem 4.13 shows that maximal operators of $\mathbf{X}$-valued tree martingale transforms are norm-bounded in $L_{p}(\mathbf{X})$ provided $\mathbf{X}$ is a $U M D$ space.

\section{ACKNOWLEDGEMENT}

The authors would like to thank the referees for their help and detailed comments. 


\section{REFERENCES}

[1] A. Benedek and R. Panzone, The spaces $L^{p}$ with mixed norm, Duke Math. J. 28(1961), 301-324. MR23:A3451

[2] J. Bourgain, Some remarks on Banach spaces in which martingale difference sequences are unconditional, Ark. Math. 21(1983), 163-168. MR85a:46011

[3] D. L. Burkholder, Martingale transforms, Ann. Math. Statist. 37(1966), 1494-1504. MR 34:8456

[4] D. L. Burkholder, A geometrical characterization of Banach spaces in which martingale difference sequences are unconditional, Ann. Prob. 9(1981), 997-1011. MR83f:60070

[5] D. L. Burkholder, Boundary value problems and sharp inequalities for martingale transforms, Ann. Prob. 12(1984), 647-702. MR86b:60080

[6] S. Fridli and F. Schipp, Tree-martingales, Proc. 5th Pannonian Symp. on Math. Stat., Visegrád, Hungary(1985), 53-63. MR89j:60064

[7] J. Gosselin, Almost everywhere convergence of Vilenkin-Fourier series, Trans. Amer. Math. Soc. 185(1973), 345-370. MR 50:5369

[8] T. J. He and Y. L. Hou, Some inequalities for tree martingales, Acta Mathematicae Applicatae Sinica (English Series) Vol. 21, No. 4(2005), 671-682. MR2006h:60074

[9] T. J. He, Y. X. Xiao and Y. L. Hou, Inequalities for vector-valued tree martingales, Proceeding of International Conference on Functional Space Theory and Its Applications, London, Research Information Ltd UK, 2004, 67-75.

[10] T. J. He, Y. L. Hou and Y. Shen, Vector-valued tree martingales and some inequalities for vector-valued tree martingales, Acta Mathematica Hungarica (submitted to).

[11] R. L. Long and P. D. Liu, Real interpolation of B-valued regular Martingale spaces, Chinese Annals of Mathematics 14A: 2(1993), 152-158. (in Chinese) MR94j:60088

[12] F. Schipp, Pointwise convergence of expansions with respect to certain product systems, Anal. Math. 2(1976), 65-76. MR54:3281

[13] F. Schipp, Universal contractive projections and a.e. convergence. Probability Theory and Applications, Essays to the Memory of József Mogyoródi. Eds.: J. Galambos, I. Kátai. Kluwer Academic Publishers, Dordrecht, Boston, London, 1992, 47-75. MR95c:42040

[14] E. M. Stein and G. Weiss, Introduction to Fourier analysis on Euclidean spaces, Princeton University Press, Princeton, New Jersey, 1971. MR46:4102

[15] F. Weisz, Martingale Hardy spaces and their applications in Fourier analysis, Lecture Notes in Mathematics, Vol. 1568, Springer, Berlin, 1994. MR96m:60108

Department of Control Science and Engineering, Huazhong University of Science and Technology, Wuhan 430074, People's Republic of China

Current address: College of Mathematics and Computer Science, Fuzhou University, Fuzhou 350002, People's Republic of China

E-mail address: hetongjun@fzu.edu.cn

Department of Control Science and Engineering, Huazhong University of Science and Technology, Wuhan 430074, People's Republic of China

E-mail address: lhfu@hust.edu.cn 